\title{
Search for Evidence of Source Event Grouping Among Ureilites
}

\author{
S.P. BEARD ${ }^{1 *}$ and T.D. SWINDLE ${ }^{1}$ \\ ${ }^{1}$ University of Arizona, Lunar and Planetary Laboratory, 1629 E University Blvd \\ Tucson, AZ 85721, USA \\ *Corresponding author. E-mail: spbeard@lpl.arizona.edu
}

\begin{abstract}
We use cosmic-ray exposure (CRE) ages of ureilites, combined with magnesium numbers of olivine, and oxygen isotopes, to search for evidence of specific source events initiating exposure for groups of ureilites. This technique can also be used to investigate the heterogeneity of the body from which the samples were derived. There are a total of 39 ureilites included in our work, which represents the largest collection of ureilite CRE age data used to date. Although we find some evidence of possible clusters, it is clear that most ureilites did not originate in one or two events on a homogeneous parent body.
\end{abstract}

\section{Introduction:}

Ureilites are ultramafic achondrites that might represent the mantle of a large single asteroid that was stratified in olivine composition, the ureilite parent body (UPB) (Goodrich, 1992; Warren et al., 1992; Goodrich et al., 2004). It is difficult to model and determine the petrogenesis of the UPB due to conflicting igneous and primitive characteristics (Goodrich, 1992; Mittlefehldt et al., 1998). Igneous properties include bulk element chemistry, mineral chemistry, and petrography suggesting that they are products of a high degree of igneous fractionation on a differentiated body (Goodrich, 1992). Primitive characteristics of ureilites include their variable oxygen isotopes (Clayton and Mayeda, 1988), high trace siderophile element abundances, and trapped noble gases of chondritic abundance with a fractionated, planetary-like, pattern (Mittlefehldt et al., 1998) that are unlikely to survive significant geologic processing. Therefore, it is hard to make a clear distinction as to whether ureilites represent primitive or differentiated materials, and whether they can be linked to a common heterogeneous source using known isotopic and mineralogical data.

Our collection of ureilites is dominated by the main group (or monomict) ureilites, thought to be mantle residues. Petrologic models predict that complementary melt rocks, which are not present in the meteorite collection, exist (referred to as the missing basalts). A subclass of the ureilites consists of polymict, fragmental breccias (e.g., Berkley et al., 1980; Goodrich et al., 2004; Downes et al., 2008) that are inherently heterogeneous on the scale of thin sections (Rai et al., 2003). These polymict ureilites contain materials spanning the same range of magnesium to iron ratios in olivine (usually given as Fo, defined as $100 *$ molar $\mathrm{Mg} /(\mathrm{Mg}+\mathrm{Fe}))$ as monomict ureilites, suggesting both classes derive from the same source (Goodrich et al., 2004; Downes et al., 2008). Polymict breccias are thought to be the regolith material formed on or near the surface of the original UPB or its offspring bodies and contain a few percent feldspathic clasts that are considered to be representative of the missing basalt (Warren and Kellemeyn, 1989; Cohen et al., 2004; Goodrich et al., 2004; Downes et al., 2004). Goodrich et al., (2004) suggest that delivery 
of ureilites to Earth is not from impacts on the UPB, but instead from offspring bodies. They argue that a common pressure and temperature history evident in monomict ureilites suggests that they derived from a common body, which later experienced very rapid cooling with a sudden drop in pressure. This could be a result of deep excavation from a major impact, with ejected debris reassembling into one or several offspring bodies (Goodrich et al., 2002). There is a continuous range in Fo among ureilites, with a peak around $\sim 79$. Goodrich et al. (2004) argue that a significant portion of collected ureilite material derived from similar depths in the UPB which, after initial break up, reassembled from this subsample of ejected materials (with Fo 79 ) to form one, or several, offspring bodies that could be the proximate parent body of ureilite meteorites. Offspring bodies are described as reassembled debris of $\sim$ meter sized monomict materials with a range of Fo's that have a prevalence of Fo $\sim 79$. Alternatively, the ejected material could have reaccreted back onto the UPB as a rubble pile (Warren and Kallemeyn, 1989). In this model, either for a rubble pile on the UPB or the creation of offspring bodies, polymict ureilites represent the regolith (Fig. 1) that overlies the main group ureilite material and is developed after reassembly (Goodrich et al., 2004).

Recent work by Goodrich et al. (2015) suggests a common history that links all ureilites to a single Ureilite Daughter Body (UDB) that formed after the initial UPB breakup. They explain the high degree of heterogeneity and foreign clast content of the Almahata Sitta polymict ureilite (Horstmann and Bischoff 2014) by suggesting it is the outer regolith material of a common UDB, which is also the source of the polymict ureilites with less foreign material (deep regolith of UDB) and the main group ureilites (interior of UDB), that have foreign clasts (enstatite, ordinary, and Rumuruti-type chondrites) implanted at low velocities. They suggest either a single break up of the UDB at $46 \mathrm{Ma}$ or older with subsequent collisions producing smaller fragments, or a cratering event at $46 \mathrm{Ma}$ followed by UDB break up at $20 \mathrm{Ma}$.

Cosmic-ray exposure (CRE) ages measure the interval of time since a meteorite departed from its last (proximate) parent body due to an impact or break-up event. Clusters of ages, measurements from multiple samples that yield the same CRE age, within a meteorite group can signify major impact events in the Main Asteroid Belt or among Near-Earth Asteroids. For example, two major events on the H-chondrite parent body are clearly seen in the large clustering of CRE ages (Graf and Marti, 1995). Discussion of clustering in other ordinary chondrites' CRE data can be found in Marti and Graf, 1992. The CRE ages of lunar and martian meteorites have been used to look for common source events (Warren, 1994). Clustering in CRE ages from three types of meteorites, the howardites, eucrites, and diogenites (HED) (e.g., Eugster and Michel, 1995) lends support to the idea that these meteorites come from a single parent body. These clusters are interpreted as representing the times of major collisions on their parent body (Eugster, 2003), since a large impact could break apart material from each of the three classes (HED) at the same time. If it is assumed that the ureilites come from a single daughter body, an impact could break apart material from different heterogeneous or homogeneous regions (Warren and Kallemeyn, 1989; Goodrich et al., 2002), which might be evident in their CRE data. 

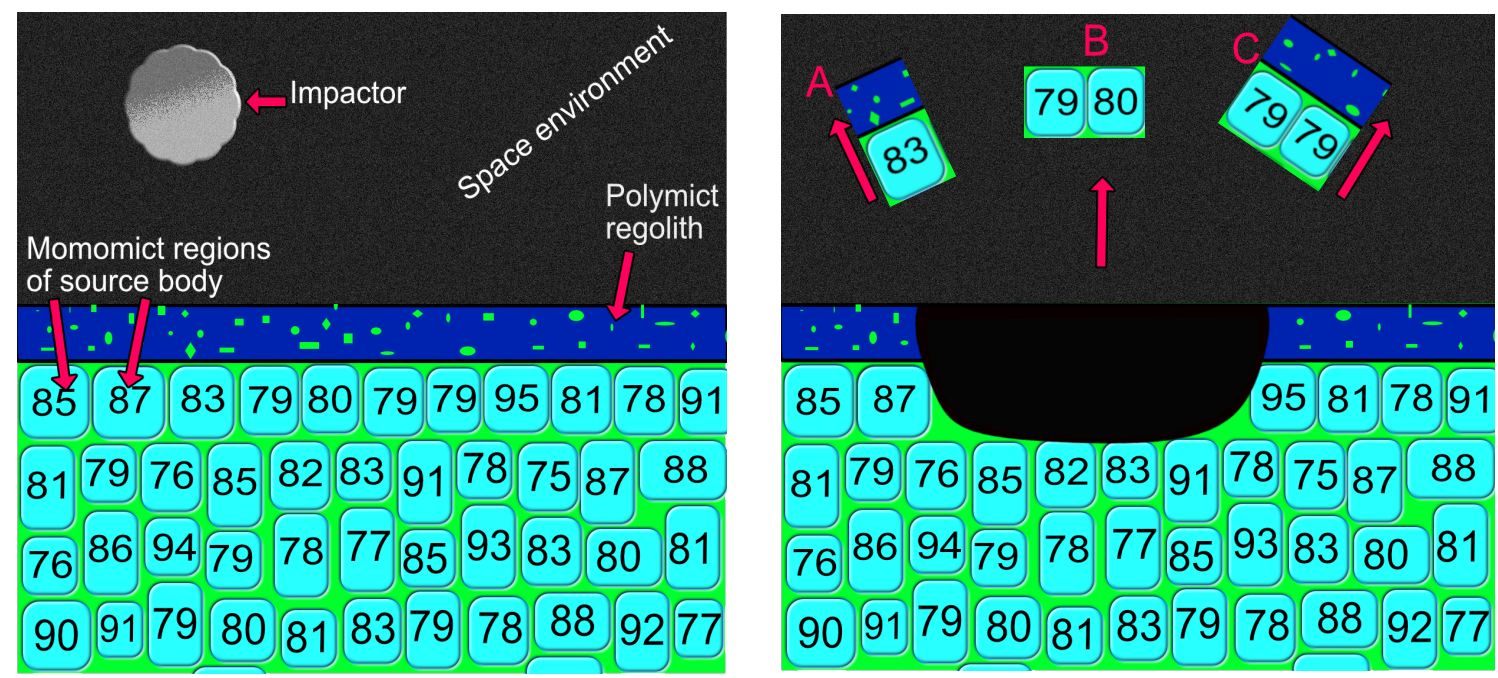

Figure. 1. (Left) Pre Impact Disruption: Possible schematic of the UPB's petrologic structure (after Goodrich et al., 2004). After catastrophic disruption, material either reaccreted onto the UPB or formed offspring bodies (UDB). In either case, the post-disruption material may have 'monomict' main group ureilite material underlying polymict material on top. (Right) Post Impact Disruption: Excavation of material from the UDB as a result of an impact could result in a number of different mixtures. A) Part of the material could be ejected as a single, whole, monomict piece with the above polymict material still attached. B) Some of the ejected material may not contain any polymict/regolith, and could be multiple monomict domains of different, or similar, Fo. C) Alternatively, some of the released material might be a mixture of multiple monomict domains with the regolith still attached. The analysis in this work would assign scenario A to be a heterogeneous grouping, with monomict and polymict samples. Scenario B would be classified as homogeneous (if the Fo are the same in addition to matching oxygen isotopes) or heterogeneous (if the Fo of the multiple monomict regions are different) and would not contain polymict samples. This scenario would also be suitable to describe a larger impact where deeper material that has no contact to the polymict region would be released. Scenario C would be considered heterogeneous with polymict and monomict samples present.

Another example of CRE age clustering can be seen in the acapulcoites and lodranites (Fig. 2), which essentially show a single age cluster (Weigel et al., 1999, Eugster et al., 2006). CRE studies for the ureilite group (Rai et al., 2003) suggest possible groupings around 1 and $10 \mathrm{Ma}$, but Rai et al. (2003) based their analysis on CRE data alone. We suggest comparison with other key properties they did not consider to provide further constraints and increase confidence in conclusions.

The goal of this work is to examine CRE ages for evidence of clusters that may improve our understanding of the origin of ureilites. We investigate possible relationships of CRE ages with other parameters and comment on how they might relate to the structure and heterogeneity of the proximate ureilite source body (Fig. 1). This will be done by comparing oxygen isotopic ratios $\left(\Delta^{17} \mathrm{O}\right)$ and the ratios of magnesium to iron in olivine (Fo) with their corresponding CRE ages to look for evidence of possible common source impact events. Although $\Delta^{17} \mathrm{O}$ and Fo tend to correlate (Clayton and Mayeda 1988; 1996; Rumble et al., 2010), an impact into a homogeneous area (Fig. 1) would be expected to produce a very tight cluster in both parameters.

Since ureilites with similar $\mathrm{O}$ isotope compositions and Fo would imply a genetic relationship, one might expect clusters of CRE ages to have similar properties if they are in fact genetic and from homogenous material. We will refer to the situation where a group of meteorites agree in all three variables, CRE age, $\Delta^{17} \mathrm{O}$, and Fo, as a 
homogeneous group or cluster, perhaps reflecting an impact into a homogeneous body or homogeneous region of a body. If the CRE ages agree, but at least one of the other variables does not, it is possible that this represents an impact into heterogeneous material. In either case described above, it cannot be unambiguosly determined if CRE clusters represent coincidental source events into different bodies or different impacts on the same heterogeneous body. If the UPB was completely disrupted and reaccreted, as might be suggested by the extremely heterogeneous Almahata Sitta breccia (Bischoff et al., 2010; Goodrich et al., 2015), CRE age clusters might be independent of petrogenetic properties, and we would expect more heterogeneous clusters. If the proximate parent body of the collection of ureilites is in fact as described in Figure 1 (note different excavation possibilities), what would the resulting debris from an impact look like? It is reasonable to postulate that an impact could break apart main group ureilite material (with a single Fo) along predefined fracture/weak points, which would then be launched simultaneously (i.e., homogeneous cluster). Alternatively, an impact could excavate ureilite materials composed of various Fo (i.e., a heterogeneous cluster).

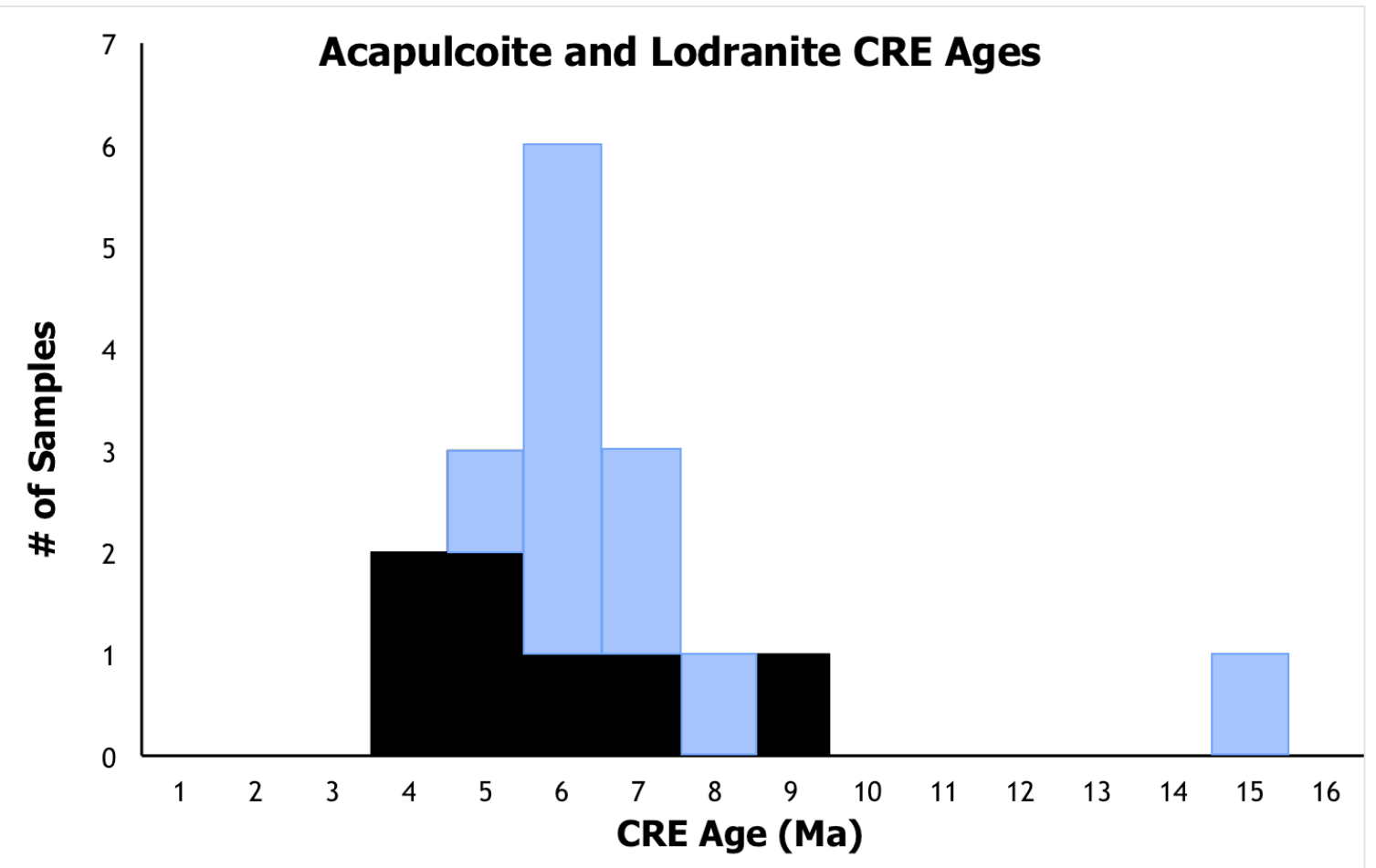

Figure 2. Histogram of CRE ages for acapulcoites and lodranites, showing a tight clustering of ages. Black regions correspond to acapulcoites while blue regions are from lodranites. Data from Weigel et al. 1999.

\section{Methods:}

CRE $\left({ }^{21} \mathrm{Ne}\right)$ ages were calculated for most of the ureilites by using $\mathrm{Ne}$ isotopic measurements from Okazaki et al. (2003), Rai et al. (2003), and Schultz and Franke (2004); along with production rates and age formulations presented by Hohenberg et al. (1978) and Wieler (2002), respectively. Production rates from Hohenberg et al. (1978) were adjusted to $4 \pi$ exposure by multiplying the reported rate by a factor of two. Shielding effects are important to consider when determining an appropriate production rate of cosmogenic isotopes. The cosmogenic ${ }^{21} \mathrm{Ne} /{ }^{22} \mathrm{Ne}$ ratio of bulk samples vary by 
only $\sim 10 \%$. This corresponds to a shielding range of $20-100 \mathrm{~g} / \mathrm{cm}^{2}$, which changes the possible production rates by up to $\sim 15 \%$. Shielding of $40 \mathrm{~g} / \mathrm{cm}^{2}$, near the peak in production rate, was assumed for all samples. While these corrections are imperfect, they are self-consistent. Cosmogenic ${ }^{21} \mathrm{Ne}$ was calculated assuming $\left({ }^{21} \mathrm{Ne} /{ }^{22} \mathrm{Ne}\right)$ trapped $=0.3$ and $\left({ }^{21} \mathrm{Ne} /{ }^{22} \mathrm{Ne}\right)_{\text {cosmogenic }}=0.9$ as described in Rai et al. (2003). When calculating the ${ }^{21} \mathrm{Ne}$ production rate, literature values were used for each sample's chemical composition ( $\mathrm{Na}$, $\mathrm{Mg}, \mathrm{Al}, \mathrm{Si}, \mathrm{Ca}$, and $\mathrm{Fe}$ ) when available. For samples whose chemical composition could not be found, an average of the remaining ureilites was used as a representative composition (Table A2, Rai et a1., 2003 and references therein). Using an average chemical composition is reasonable, since CRE ages calculated using the range of compositions had little effect on the outcome. Multiple CRE ages have been calculated for samples that had multiple isotopic measurements available, yielding an average exposure age with the uncertainty expressed as the standard deviation. The standard deviations varied from $5 \%$ to $21 \%$, with an average of $13 \%$, with the exception of one sample with only two measurements that differed by $44 \%$. For samples for which only one measurement was available, we assumed a $20 \%$ uncertainty, a conservative value based on the standard deviation among other samples that have data from multiple measurements. The ureilite Almahata Sitta was also added to this study (using the average of 6 measurements from Almahata Sitta ureilite samples measured by Welten et al. 2010), resulting in a Ne-based CRE age of Almahata Sitta (15.8 Ma) that agrees with the Ne-based CRE age reported by Welten et al. (2010) to within 10\% (although they preferred a Ne-Al age of $19 \mathrm{Ma}$ ). Eight measurements in Table 1 denoted with an asterisk come directly from an abstract by Park et al. (2014). For these samples we used the CRE ages reported in the abstract, since isotopic data have not been published. Since we did not recompute these ages, they do not contain the same systematic uncertainties as our calculations, however, we feel it is valid to include them in our analysis since identical results were obtained independently for common samples. In all, we have tabulated 39 ureilite CRE ages.

CRE ages were plotted against literature values of oxygen isotopes $\left(\Delta^{17} \mathrm{O}\right)$ and forsterite content of olivine (Fo), i.e. $100 *$ molar $\mathrm{Mg} /(\mathrm{Mg}+\mathrm{Fe}$ ) (Clayton and Mayeda, 1988, 1996; Goodrich, 1999; Kita et al., 2004; Table 23 of Mittlefehldt et al., 1998; Weber and Bischoff, 1998) to find if there are any groupings in all three variables. Uncertainties for Fo and $\Delta \Delta^{17} \mathrm{O}$ either reflect the uncertainty or deviation (specifically important for polymict ureilites where the entire range was used) in the literature or were assigned a conservative value of \pm 2 when uncertainties could not be found for Fo. Oxygen isotopic values could not be found for every sample in this study - more $\Delta^{17} \mathrm{O}$ data would increase the effectiveness of this type of analysis if included in the future.

If CRE ages, Fo, and $\Delta^{17} \mathrm{O}$ values agree, it is considered to be a homogenous cluster, potentially a homogeneous event grouping. If one or two variables disagree, the CRE cluster is classified as a heterogeneous cluster. In some cases, there are homogenous subsets within a heterogeneous cluster. Possible explanations of this include coincident impacts into homogenous material on multiple independently homogenous bodies/regions, or a single impact into heterogeneous material. Reported cluster ages are determined by taking the average exposure age in the cluster, taking the standard deviation within the group as the error. Alternatively, the error could be calculated by taking the root mean square of the CRE errors, which does not change results 
significantly. We recognize there are systematic uncertainties not included in this work that are larger than what is reported for individual measurements.

Table 1. Key data for the 39 ureilites used in this work.

\begin{tabular}{|c|c|c|c|c|c|}
\hline Ureilite & CRE age (Ma) & CRE Error (Ma) & $\Delta^{17} \mathbf{O}$ & Fo \# & Polymict \\
\hline Acfer 277 & 0.53 & 0.1 & -1.06 & 83.2 & \\
\hline Allan Hills (ALHA) 77257 & 9.10 & $1.8^{\wedge}$ & -0.9 & 84.9 & \\
\hline Allan Hills (ALH) 78019 & 0.09 & 0.01 & -0.83 & 76.2 & \\
\hline Allan Hills (ALHA) 81101 & 10.86 & 2.6 & -0.23 & 79 & \\
\hline Allan Hills (ALH) 82130 & 1.51 & 0.3 & -2.53 & 95 & \\
\hline Almahata Sitta & 15.80 & 0.9 & -1.22 & 88.5 & \\
\hline Asuka (A-) 881931 & 3.85 & 1.9 & - & 76 & \\
\hline Dar al Gani (DaG) 084 & 19.10 & 3.8 & - & 79.1 & \\
\hline Dar al Gani (DaG) 319 & 21.67 & 2.2 & - & 79 & $x$ \\
\hline Dar al Gani (DaG) 340 & 8.74 & 0.1 & -1.2 & 79.9 & \\
\hline Dingo Pup Donga & 4.24 & $0.8^{\wedge}$ & -1.29 & 84 & \\
\hline Dyalpur & 9.09 & 1.9 & -1.57 & 84 & \\
\hline Elephant Moraine (EET) 83225* & 2.77 & 0.6 & - & 87 & \\
\hline Elephant Moraine (EET) 83309 & 38.01 & 7.5 & -1.2 & 82 & $x$ \\
\hline Elephant Moraine (EET) 87511* & 3.30 & 0.7 & - & 85 & \\
\hline Elephant Moraine (EET) 87517* & 7.77 & 1.6 & - & 92 & \\
\hline Elephant Moraine (EET) 87720 & 7.34 & 0.3 & -0.53 & 83 & $x$ \\
\hline Goalpara & 20.50 & 0.6 & -0.4 & 78.6 & \\
\hline Graves Nunataks (GRA) 95205* & 10.09 & 2.1 & - & 79 & \\
\hline Graves Nunataks (GRA) 98032* & 15.32 & 0.8 & - & 75 & \\
\hline Grosvenor Mountains (GRO) 95575* & 5.22 & 1.0 & - & 78.7 & \\
\hline Hajmah (a) & 1.10 & $0.2^{\wedge}$ & - & 85 & \\
\hline Hammadah al Hamra $(\mathrm{HaH}) 064$ & 0.76 & $0.2^{\wedge}$ & -0.74 & 78 & \\
\hline Haverö & 19.29 & 1.1 & -0.52 & 79 & \\
\hline Kenna & 24.74 & 5.5 & -1.02 & 79 & \\
\hline Lahrauli & 15.42 & 0.6 & - & 79 & \\
\hline Lewis Cliff (LEW) 85328 & 11.52 & $2.3^{\wedge}$ & -0.57 & 80 & \\
\hline Meteorite Hills (MET) 01083* & 7.17 & 1.4 & - & 91.9 & \\
\hline Meteorite Hills (MET) 01085* & 25.13 & 5.0 & - & 90 & \\
\hline Nilpena & 9.02 & 1.2 & -1.39 & 80 & $\mathrm{x}$ \\
\hline North Haig & 0.13 & $0.03^{\wedge}$ & -0.97 & 84 & $x$ \\
\hline Novo-Urei & 5.90 & 0.8 & -0.99 & 79 & \\
\hline Pecora Escarpment (PCA) 82506 & 2.81 & $0.6^{\wedge}$ & -0.88 & 78.3 & \\
\hline Reckling Peak (RKP) A80239 & 21.89 & 4.3 & -1.12 & 84 & \\
\hline Roosevelt County (RC) 027 & 2.11 & $0.4^{\wedge}$ & -1.08 & 79.4 & \\
\hline Sahara 98505 & 13.20 & 2.5 & - & 81.2 & \\
\hline Yamato (Y-) 74123 & 4.01 & 1.0 & -0.81 & 79.2 & \\
\hline Yamato (Y-) 790981 & 11.66 & $2.3^{\wedge}$ & -0.52 & 77.5 & \\
\hline Yamato (Y-) 791538 & 2.64 & 0.5 & -1.9 & 91.8 & \\
\hline
\end{tabular}

CRE age calculations are based on data from Okazaki et al., (2003), Rai et al., (2003) and Schultz and Frank, (2004). Oxygen isotopes and forsterite numbers were compiled from Clayton and Mayeda, (1988,1996); Goodrich, (1999); Kita et al., (2004); Mittlefehldt et al., (1998): see Table 23; Weber and Bischoff, (1988). CRE ages from meteorites denoted by an asterisk (*) are from Park et al., (2014). Cases where ages were determined from one measurement were assigned a $20 \%$ error and are denoted by a $\left(^{\wedge}\right)$.

For the five polymict ureilites, we would expect the CRE ages of the various clasts to agree, unless pre-compaction exposure occurred. We are not aware of any evidence of 
pre-compaction exposure within ureilites, although we cannot rule it out. For these meteorites, we would expect the $\mathrm{O}$ isotopes to represent an average over the materials analyzed, while we use the range of Fo values measured.

\section{Results:}

CRE ages are reported in Table 1, and agree well with prior work where comparisons can be made (Rai et al., 2003). These ages are also shown in Figure 3 as a relative probability plot (each age is represented as a Gaussian distribution, all with the same area under the curve, so the width is proportional to the uncertainty and more precisely defined ages have narrower and taller Gaussians) and Figure 4 in the form of a histogram for easy comparison to prior publications.

In general, there is a wide range of mostly randomly distributed CRE ages, with no clear cluster identified. However, there is evidence of possible clusters, which we will discuss in order to illustrate the method of searching for and assigning clusters. Ages for potential clusters are around $<1 \mathrm{Ma}, \sim 1 \mathrm{Ma}, \sim 2-3 \mathrm{Ma}, \sim 4-5 \mathrm{Ma}, \sim 9 \mathrm{Ma}$, and $\sim 20 \mathrm{Ma}$ based on ages alone. When comparing the Fo to CRE age (Fig. 5), we add another variable for determining the likelihood of source event clusters and now see possible clusters and subclusters at $<1 \mathrm{Ma}, \sim 1 \mathrm{Ma}, \sim 3 \mathrm{Ma}, \sim 4-5 \mathrm{Ma}, \sim 9 \mathrm{Ma}, \sim 12 \mathrm{Ma}, \sim 15 \mathrm{Ma}$, and $\sim 20 \mathrm{Ma}$. Possible clusters from CRE and $\Delta^{17} \mathrm{O}$ (Fig. 6) include groups at $<1 \mathrm{Ma}, \sim 3-$ $4 \mathrm{Ma}, \sim 9-11 \mathrm{Ma}$, and $20 \mathrm{Ma}$. Final determination of possible clusters can be seen in Table 2. The data were scrutinized carefully to look for signs of age clusters but nothing stands out. The clusters briefly discussed below, and listed in Table 2, are possible. It is possible that more data, or data with higher resolution noble gas measurements or more $\mathrm{O}$ isotope measurements, would reveal more definitive evidence for clustering.

\section{Possible Homogenous Clusters:}

Combining all three sets of data (CRE, Fo, and $\Delta^{17} \mathrm{O}$ ), possible evidence of three homogenous clusters is present at $0.1 \pm 0.02 \mathrm{Ma}, 5.6 \pm 0.5 \mathrm{Ma}$, and $20.1 \pm 1.2 \mathrm{Ma}$. One main group and one polymict ureilite form the youngest cluster, $0.1 \pm 0.02 \mathrm{Ma}$. The next source event cluster at $5.6 \pm 0.5 \mathrm{Ma}$, from two main group ureilites, is missing one of the $\Delta^{17} \mathrm{O}$ values but is homogeneous in Fo and age. The last homogenous cluster is at $20.1 \pm$ 1.2 $\mathrm{Ma}$ and is formed from three main group and one polymict ureilite. Note that all homogenous parings have Fo $\sim 79$, but the two more recent groups have $\Delta^{17} \mathrm{O}$ of $\sim-1.0 \%$ o while the older event has $\Delta^{17} \mathrm{O}$ of $\sim-0.5 \%$.

\section{Possible Heterogeneous Clusters:}

Several clusters agree in CRE age and Fo, but not $\Delta^{17} \mathrm{O}$. We did not find any cases with agreement between only $\mathrm{CRE}$ age and $\Delta^{17} \mathrm{O}$. Seven heterogeneous clusters are assigned at $0.7 \pm 0.1 \mathrm{Ma}, 1.3 \pm 0.3 \mathrm{Ma}, 3.4 \pm 0.7 \mathrm{Ma}, 7.4 \pm 0.3 \mathrm{Ma}, 9.3 \pm 0.4 \mathrm{Ma}, 12.4 \pm$ 0.7 , and $15.5 \pm 0.3 \mathrm{Ma}$, four of which show possible evidence of homogenous subclusters. There are two homogeneous subclusters that form the group at $3.4 \pm 0.7 \mathrm{Ma}$. The heterogeneous cluster at $7.4 \pm 0.3 \mathrm{Ma}$ is a combination of a homogeneous subcluster and a single sample. Another distinct cluster with two possible homogeneous subclusters is evident at $9.3 \pm 0.4 \mathrm{Ma}$. The last heterogeneous cluster of this type is at $12.4 \pm 0.7 \mathrm{Ma}$. All the meteorites in these clusters are main group except for Nilpena and Elephant 


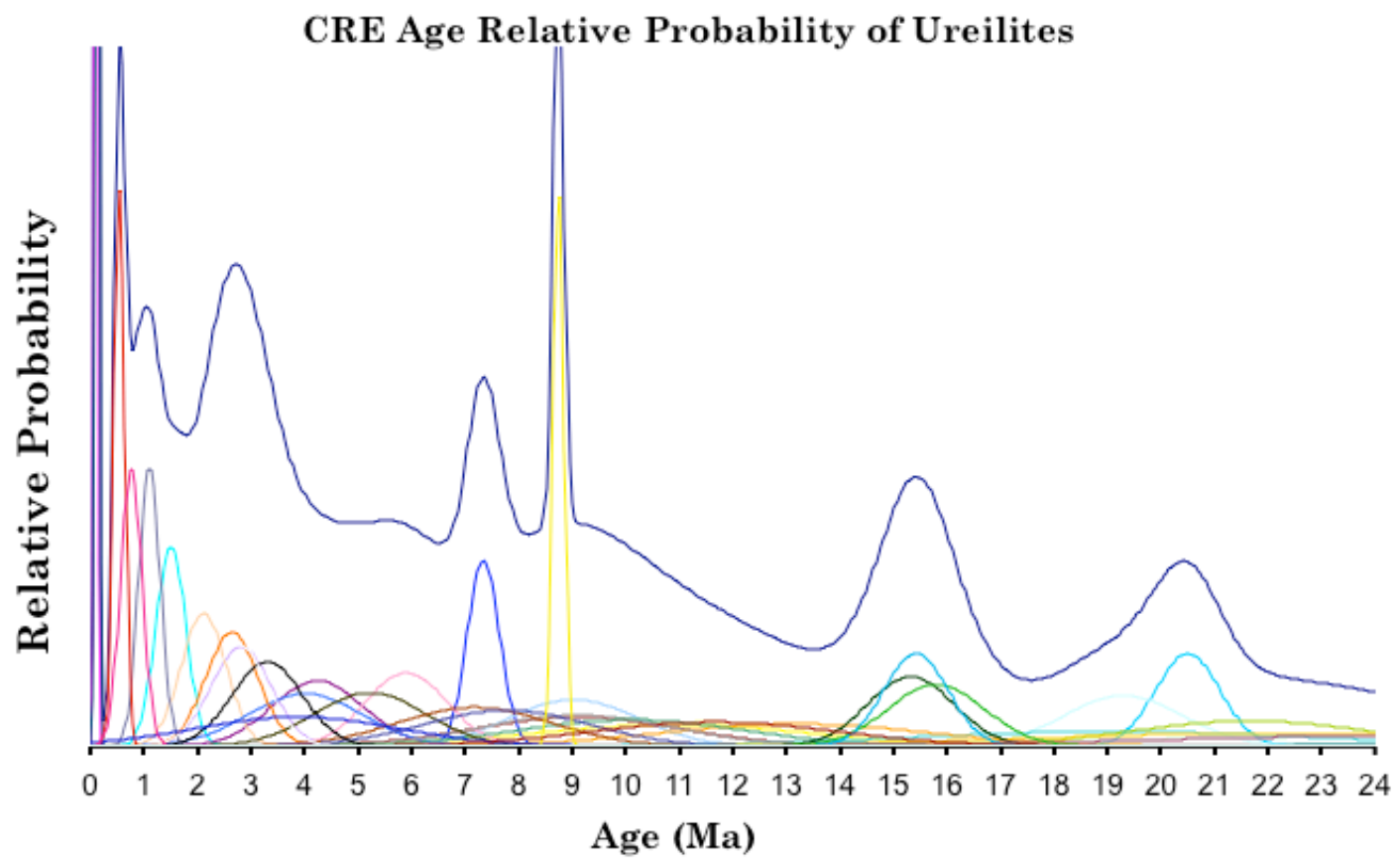

Figure. 3. Relative probability plot (ideogram) of the CRE ages of ureilites in this study. Possible groupings appear to be at $\sim 0.1, \sim 1, \sim 3, \sim 9, \sim 15$ and $\sim 20$ Ma. Values of y-axis are arbitrary units.

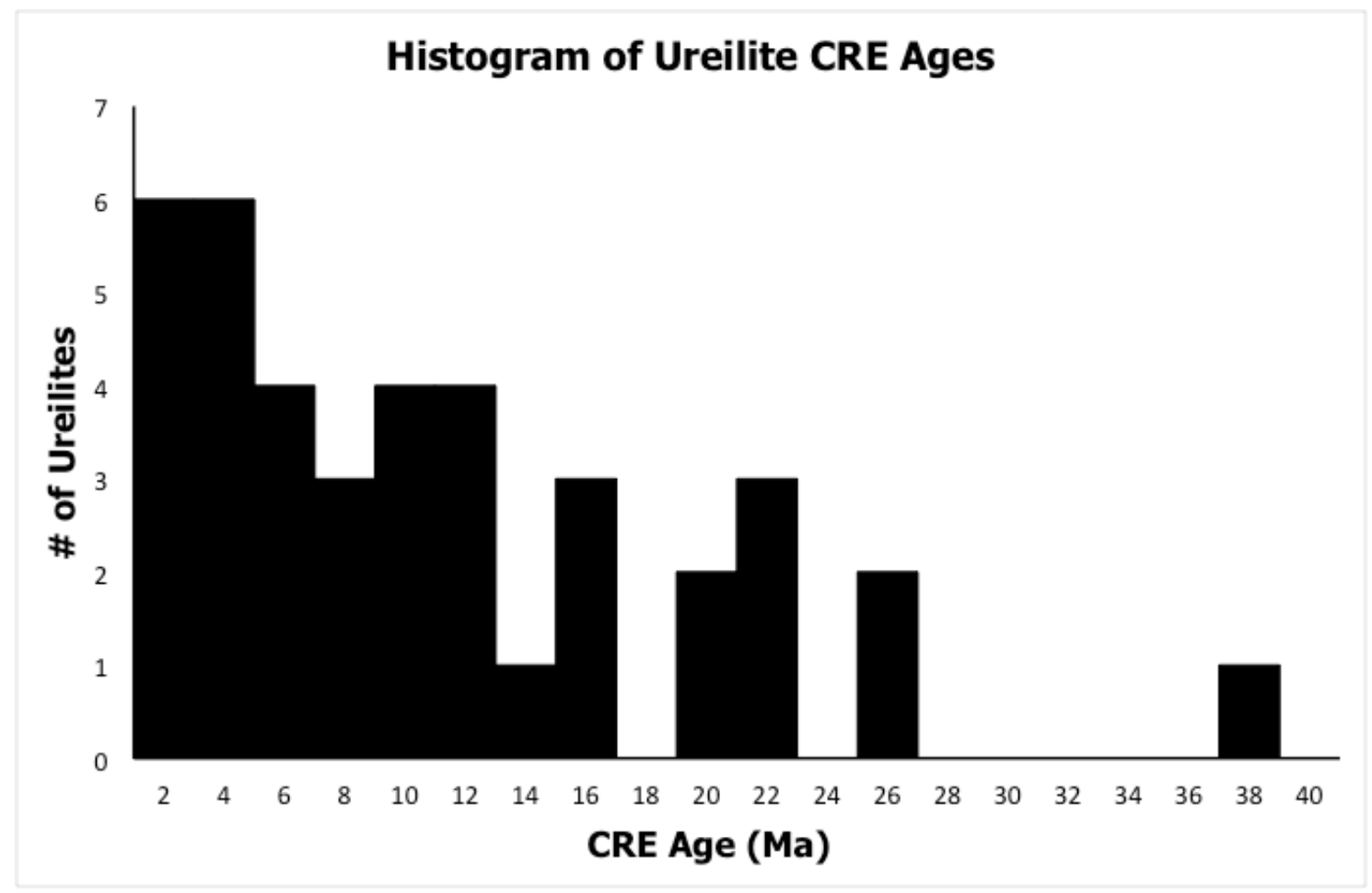

Figure. 4. Histogram of 39 ureilite CRE ages. Note that the data are much more scattered than that for the acapulcoites and lodranites in Fig. 2. Data is represented in 'bins' of 2 Ma. 


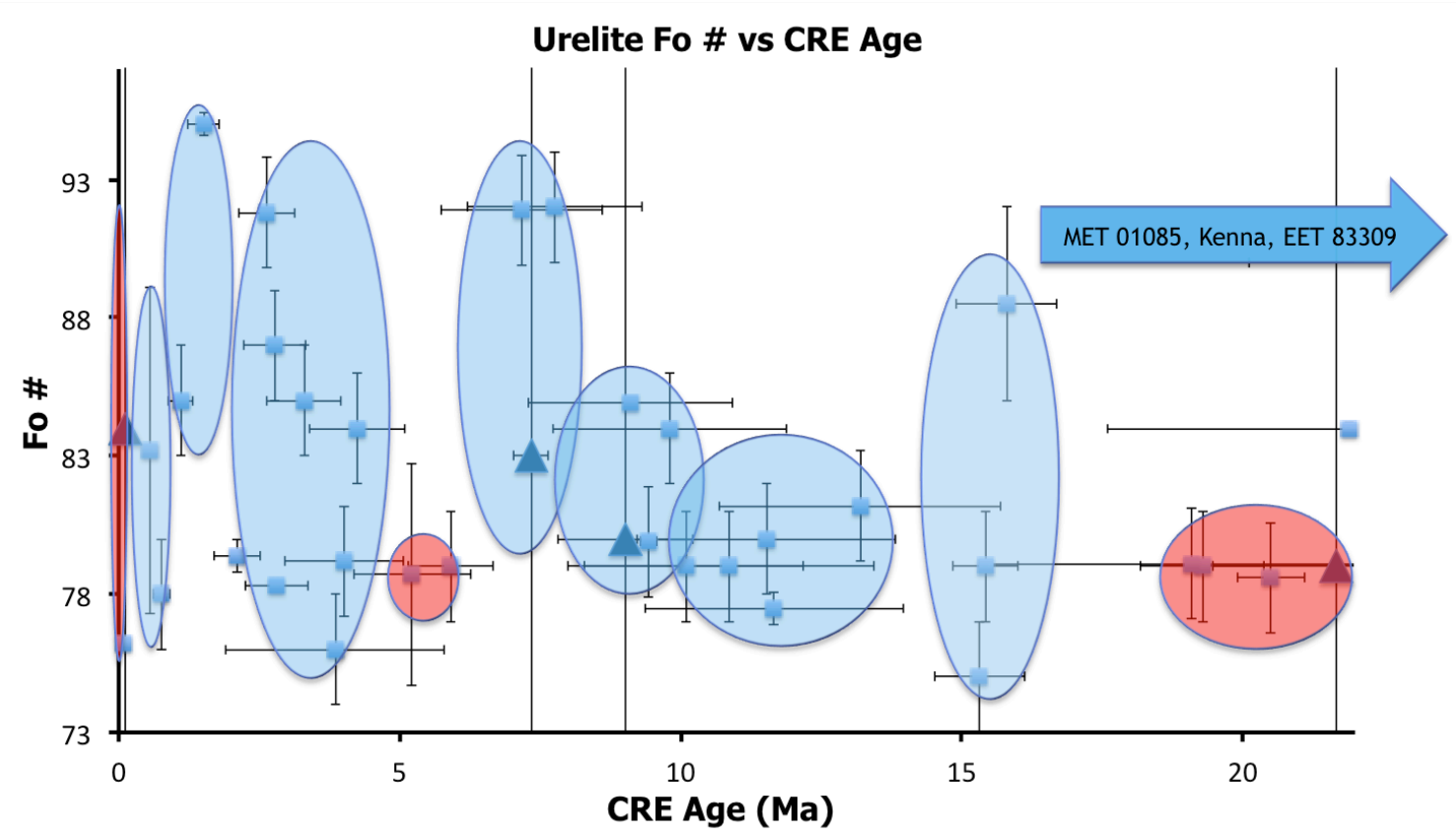

Figure. 5. Ureilite Fo vs CRE Age. This shows an additional constraint on Fig. 3 since it shows samples that have the same CRE age and Fo (within error), which might suggest these samples are material ejected from the same impact (shaded groupings). Triangles denote samples that are polymict.

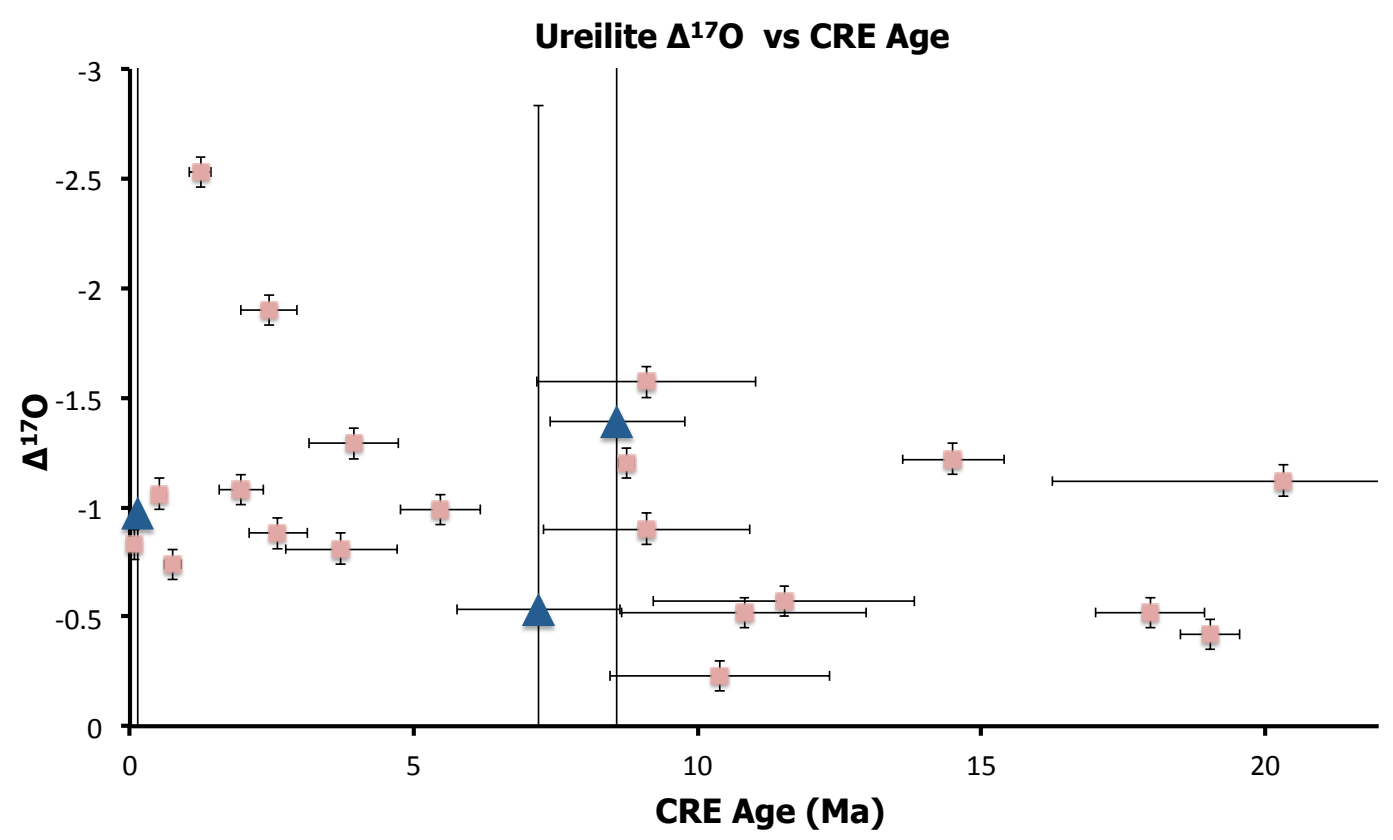

Figure. 6. Ureilite $\Delta^{17} \mathrm{O}$ vs CRE ages showing additional constraints on Figs. 3 and 5. Now samples can be evaluated to check for agreement in $\mathrm{CRE}$ age, Fo, and $\Delta^{17} \mathrm{O}$ values. Triangles denote polymict samples. 
Table 2. Possible Clusters.

\begin{tabular}{lll}
\hline Age (Ma) & \multicolumn{1}{c}{ Type } & \multicolumn{1}{c}{ Meteorites } \\
\hline $0.1 \pm 0.02$ & Homogeneous & ALH 78019, North Haig (P) \\
$0.7 \pm 0.1$ & Heterogeneous & Acfer 277, Hah 064 \\
$1.3 \pm 0.3$ & Heterogeneous & ALH 82130, Hajmah (a) \\
$3.4 \pm 0.7$ & Heterogeneous* & (A-881931, PCA 82506, Y-74123), (Dingo Pup Donga, EET 83225, EET 87511), Y-791538 \\
$5.6 \pm 0.5$ & Homogeneous (?) & GRO 95575, Novo-Urei \\
$7.4 \pm 0.3$ & Heterogeneous* & (EET 87515, MET 01083), EET 87720 (P) \\
$9.3 \pm 0.4$ & Heterogeneous* & (ALHA77257, Dylpur, (Nilpena (P), DaG 340) \\
$12.4 \pm 0.7$ & Heterogeneous* & (LEW 85328, Sahara 98505, Y-790981), (ALHA81101, GRA 95205) \\
$15.5 \pm 0.3$ & Heterogeneous & Almahata Sitta, GRA 98032, Lahrauli \\
$20.1 \pm 1.2$ & Homogeneous & DaG 084, DaG 319 (P), Goalpara, Haverö \\
\hline
\end{tabular}

Heterogeneous clusters that have an asterisk signify the presence of homogeneous subclusters, which are listed in parenthesis. The homogeneous cluster at 5.6 Ma has a question mark (?) since it is homogenous in Fo and age, but oxygen data is lacking. Polymict ureilites are denoted by a "P" in parentheses. Details about individual meteorites are in Table 1.

Moraine 87720, which are polymict and are each in different groupings. Half of these groups contain samples that have an Fo $\sim 79$.

There are three possible event clusters that are heterogeneous with no evidence of homogeneous subclusters. The youngest such cluster is at $0.7 \pm 0.1 \mathrm{Ma}$, followed by a heterogeneous cluster at $1.3 \pm 0.3 \mathrm{Ma}$. The last cluster of this type is at $15.5 \pm 0.3 \mathrm{Ma}$, and includes Almahata Sitta. At least one sample in each of these clusters has Fo of 79.

\section{Discussion:}

The ureilites do not show significant evidence for groupings, certainly not at the level of, for example, the acapulcoites/lodranites (Fig. 2). The CRE data for the ureilites are rather scattered, but we will discuss possible groupings that as a whole are unconvincing but may in fact hint at weakly developed clusters. It is clear that most ureilites did not originate from one or two impact events.

There are two basic ways to approach the classification of these clusters; one is to look for homogeneous clusters, i.e., look for any clusters that share agreement in CRE age, Fo, and $\Delta^{17} \mathrm{O}$ values. Alternatively (see results section), one can look at age clusters first, and then determine how homogenous or heterogeneous the age cluster is as a whole. This work shows possible evidence of three homogenous clusters, which if each represents a single source event, suggests a homogeneous source body, or a source body that is at least regionally (or locally) homogenous. The cluster at $5.6 \pm 0.5 \mathrm{Ma}$ only has one known oxygen value, so there is a possibility that this is a heterogeneous cluster. The cluster at $20.1 \pm 1.2 \mathrm{Ma}$ includes one polymict ureilite and three main group ureilites. It was decided to include all four samples into this source cluster due to the agreement in age and Fo, despite the fact that no $\Delta^{17} \mathrm{O}$ data could be found for two of the meteorites in this cluster. The Fo for the homogeneous clusters are all $\sim 79$ (not surprising given the prevalence of Fo $\sim 79$ among ureilites), which, combined with the fact that there are only two distinguishable oxygen isotope values, might suggest that the source body is dominated by Fo $\sim 79$ material.

However, there are many more possible heterogeneous clusters identified in this work, suggestive of a heterogeneous source. Cases where there is a cluster in ages with heterogeneous Fo and/or $\Delta^{17} \mathrm{O}$ could represent a single impact into a heterogeneous source, or alternatively two or more separate impact events on different bodies around the 
same time. Classification of homogeneous subclusters within a cluster was determined based on the natural segregation of the Fo among these clusters (i.e., the cluster at 3.4 Ma, the most likely example of a cluster, has two sets of three samples that have the same Fo, making it unclear if this set represents an impact into heterogeneous material or different impacts at roughly the same time), as well as differences in $\Delta^{17} \mathrm{O}$ values (though some data are lacking). Finally, the homogenous clusters previously discussed (0.1 Ma, 5.6 Ma, and 20.1 Ma) could in fact represent a subset of a heterogeneous cluster that we do not yet have evidence of. Several samples in this study lack oxygen isotopic data (Table 1), which if known would help better determine the classification scheme used in this work. Two heterogeneous clusters $(7.4 \pm 0.3 \mathrm{Ma}$ and $9.3 \pm 0.4 \mathrm{Ma})$ have a sample that has an Fo that overlaps with both clusters. A decision was made to include it in one of the clusters since inclusion in one or the other does not significantly change results.

To be clear, a heterogeneous cluster does not exclude that these samples were liberated from the same impact event, but could reflect the degree of homogeneity of the UDB or precursor body. For example, if a cluster agrees in CRE age, and one sample has Fo of 79 and another sample has Fo of 84, they could be two separate and neighboring 'monomict' regions ejected from the same impact (Fig. 1). Depending on the true scale of heterogeneity of the ureilite source body, the case of a small disagreement in oxygen isotopes does not exclude that these samples are from the same event. If the two sets of meteorites are from the same event, the heterogeneity in isotopes and chemistry may be due to the fact that they were located far apart during a large collision. Further, if the UPB was completely disrupted and reaccreted, CRE age clusters might be independent of petrogenetic properties, and we would expect heterogeneous clusters or locally homogeneous clusters, which is supported by this work.

The models of offspring bodies (Goodrich et al., 2002, 2004) suggest the possibility that rubble of polymict material overlays heterogeneous patches of main group ureilite material. Of the possible source event parings suggested in this work, three events have a mixture of main group and polymict ureilites, while the seven other tentative events contain only main group ureilites. If these proposed events are truly from a small number of impacts on the ureilite source(s), then the cases in which there are both polymict and main group ureilites means these events potentially have material from both the surface and underlying layers that survived the impact and journey to Earth. Alternatively, these clusters could be coincidences and are not real. Furthermore, this study includes just 39 out of over 350 individual ureilites (Meteoritical Bulletin Database), so much more insight into possible source paired events could be achieved from further study using this approach. Goodrich et al. (2015) suggest the possibility of the break up of the UDB at $\sim 20 \mathrm{Ma}$, consistent with our oldest proposed cluster.

\section{Conclusions:}

Ureilites are a heterogeneous group of achondrites with both primitive and differentiated characteristics. Goodrich et al. (2004) proposed a model that has polymict material overlaying main group (a.k.a. monomict) material of various Fo. We do not see evidence of a large $\sim 50$ Ma break up, unless smaller pieces were larger than multi-meter sized, but we do see evidence of a possible break up event $20 \mathrm{Ma}$. CRE ages of ureilites generally show a wide distribution and show that samples do not come from just one or two major impact events. Several possible clusters have been further constrained by 
considering Fo and oxygen isotopic data. Although no ureilite clusters or events can be confirmed, the technique for classification of clusters and its implications are discussed. This correlation of data has produced possible homogenous clusters at $\sim 0.1 \pm 0.02 \mathrm{Ma}$, $5.6 \pm 0.5 \mathrm{Ma}$, and $20.1 \pm 1.2 \mathrm{Ma}$, which suggest some degree of homogeneity in the ureilite source(s), at least regionally. Two of these clusters contain both polymict and main group ureilites, which may hold interesting consideration for heterogeneity, impact parameters, and the structure of the ureilite source. Signs of seven heterogeneous clusters have been identified, that if real, could represent coincidental source events into different bodies or different areas of a heterogeneous body. Rai et al. (2003) suggest possible clusters in ureilites $(\sim 1$ and $\sim 10 \mathrm{Ma})$, which we also see in our heterogeneous clusters, with the addition of more data and further chemical and isotopic constraints that can be used as a future analysis technique. Although some arguments could be made for assigning clusters, this work demonstrates that the ureilites as a whole show a wide variety among CRE ages, unlike the H-chondrites and acapulcoites/lodranites (Eugster et al., 2006). 


\section{REFERENCES}

Berkley J. L., Taylor G. J., Keil K., Harlow G. E. and Prinz M. 1980. The nature and origin of ureilites. Geochimica et Cosmochimica Acta 44:1579-1597.

Bischoff A., Horstmann M., Pack A., Laubenstein M. and Haberer S. 2010. Asteroid $2008 \mathrm{TC}_{3}$-- Almahata Sita: A spectacular breccia containing many different ureilitic and chondritic lithologies. Meteoritics and Planetary Science 45: 1638-1656.

Clayton R. N. and Mayeda T. K. 1988. Formation of ureilites by nebular processes. Geochimica et Cosmochimica Acta 52: 1313-1318.

Clayton R. N. and Mayeda T. K. 1996. Oxygen isotope studies of achondrites. Geochimica et Cosmochimica Acta 60: 1999-2017.

Cohen B. A., Goodrich C. A., Keil K. 2004. Feldspathic clast populations in polymict ureilites: Stalking the missing basalts from the ureilite parent body. Geochimica et Cosmochimica Acta 68:4249-4266.

Downes H., Mittlefehldt D. W., Kita N. T., Valley J. W. 2008. Evidence from polymict ureilites for a disrupted and re-accreted single ureilite parent asteroid gardened by several distinct impactors. Geochimica et Cosmochimica Acta 72: 48254844.

Eugster O., Michel T. 1995. Common asteroid break-up events of eucrites, diogenites, and howardites and cosmic-ray production rates for noble gases in achondrites. Geochimica et Cosmochimica Acta 59: 177-199.

Eugster O. 2003. Cosmic-ray exposure ages of meteorites and lunar rocks and their significance. Chemie der Erde 63: 3-30.

Eugster O., Herzog G. F., Marti K., Caffee M. W. 2006. Irradiation Records, Cosmic-Ray Exposure Ages, and Transfer Times of Meteorites. In Meteorites and the early solar system, edited by Lauretta D. S. and McSween H. Y. Jr. Tucson: The University of Arizona Press. Pp. 829-851.

Goodrich C. A. 1992. Ureilites: A critical review. Meteoritics and Planetary Science 27: 327-352.

Goodrich C. A. 1999. Are ureilites residues from partial melting of chondritic material? The answer from MAGPOX. Meteoritics and Planetary Science 34: 109119.

Goodrich C. A., Krot A. N., Scott E. R. D., Taylor G. J., Fioretti A. M., Keil K. 2002. Formation and evolution of the ureilite parent body and its offspring (abstract). Lunar and Planetary Science 33, 1379.

Goodrich C. A., Scott E. R. D., and Fioretti A. M. 2004. Ureilite breccias: Clues to the petrologic structure and impact disruption of the ureilite parent body. Chemie der Erde 64:283-327.

Goodrich C. A., Hartmann W. K., O'Brien D. P., Weidenschilling S. J., Wilson L., Michel P., and Jutzi M. 2015. Origin and history of ureilitic material in the solar system: The view from asteroid $2008 \mathrm{TC}_{3}$ and the Almahata Sitta meteorite. Meteoritics and Planetary Science 50: 782-809.

Graf T. and Marti K. 1995. Collisional history of H chondrites. Journal of Geophysical Research 100: 21,247-263.

Hohenberg C. M., Marti K., Podosek F. A., Reedy R. C. and Shirck J. R. 1978. 
Comparisons between observed and predicted cosmogenic noble gases in lunar samples. Proceedings, $9^{\text {th }}$ Lunar and Planetary Science Conference. pp. 2311-2344.

Horstmann M., Bischoff A. 2014. The Almahata Sitta polymict breccia and the late accretion of asteroid $2008 \mathrm{TC}_{3}$. Chemie der Erde 74:149-183.

Kita N. T., Ikeda Y., Togashi S., Liu Y., Morishita Y., and Weisberg M. K. 2004. Origin of ureilites inferred from a SIMS oxygen isotopic and trace element study of clasts in the Dar al Gani 319 polymict ureilite. Geochimica et Cosmochimica Acta 68: 4213-4235.

Leya I., and Masarik J. 2009. Cosmogenic nuclides in stony meteorites revisited. Meteoritics and Planetary Science 44: 1061-1086.

Marti K., and Graf T. 1992. Cosmic-ray exposure history of ordinary chondrites. Annual Reviews in Earth and Planetary Science 20. pp. 221-243.

Mittlefehldt D. W., McCoy T. J., Goodrich C. A., and Kracher A. 1998. Non-chondritic meteorites from asteroidal bodies. Ureilites. In Planetary Materials, edited by Papike J. J. Reviews in Mineralogy 36: 4,1-195. Washinton, D.C.: Mineralogical Society of America.

Okazaki R., Nakamura T., Takaoka N., and Nagao K. 2003. Noble gases in ureilites released by crushing. Meteoritics and Planetary Science. 38: 767-781.

Park J., Herzog G. F., Haba M. K., Nagao K. 2014. Exposure Ages of Ureilites: Radionuclides and Noble Gases (abstract). Lunar and Planetary Science 45, 1777.

Rai V. K., Murty S. V. S. and Ott U. 2003. Noble gases in ureilites: Cosmogenic, radiogenic, and trapped components. Geochimica et Cosmochimica Acta 67: 44354456.

Rumble D., Zolensky M. E., Friedrich J. M., Jenniskens P., Shaddad M. H. 2010. The oxygen isotope composition of Almahata Sitta. Meteoritics \& Planetary Science 45:1765-1770.

Russell S. S., Folco L., Zipfel J., Zolensky M. E., Grady M. M., Jones R., Righter K., Grossman J. N. 2004. The Meteoritical Bulletin, No. 88. Meteoritics and Planetary Science 39, A215-A279.

Schultz L. and Franke L. 2004. Helium, neon, and argon in meteorites: A data collection. Meteoritics and Planetary Science 39: 1889-1890.

Warren P. H., Kallemeyn G. W. 1989. Geochemistry of polymict ureilite EET83309, and a partially disruptive impact model for ureilite origin. Meteoritics 24, 233-246.

Warren P. H. and Kallemeyn G. W. 1992. Explosive volcanism and the graphiteoxygen fugacity buffer on the parent asteroid(s) of the ureilite meteorites. Icarus 100: $110-126$.

Warren P. H. 1994. Lunar and Martian meteorite delivery systems. Icarus 111: 338363.

Weber I. and Bischoff A. 1998 Mineralogy and chemistry of the ureilites Hammadah al Hamra 064 and Jalanash (abstract). Lunar and Planetary Science 19:1365.

Weigel A., Eugster O., Koeberl C., Michel R., Kr̈ahenb̈uhl U., Neumann S., 1999. Relationships among lodranites and acapulcoites: noble gas isotopic abundances, chemical composition, cosmic-ray exposure ages, and solar cosmic ray effects. Geochimica et Cosmochimica Acta 63:175-192.

Welten K. C., Meier M. M. M, Caffee M. W., Nishiizumi K., Wieler R., Jenniskens P., and Shaddad M. H. 2010. Cosmogenic nuclides in Almahata Sitta ureilites: 
Cosmic-ray exposure age, preatmospheric mass, and bulk density of asteroid 2008 $\mathrm{TC}_{3}$. Meteoritics and Planetary Science 45: 1728-1742.

Wieler R. 2002. Noble gases in the solar system. Reviews and Mineralogy Geochemistry 47: 21-70. 